\title{
FSS Based Circular Polarizer for High-Speed Wireless Communication at 75 GHz
}

\author{
Ghaffer Kiani \\ Department of Electrical and Computer Engineering, King Abdulaziz University, Jeddah, KSA \\ Email: gkiani@kau.edu.sa
}

How to cite this paper: Kiani, G. (2019) FSS Based Circular Polarizer for High-Speed Wireless Communication at $75 \mathrm{GHz}$. Open Journal of Antennas and Propagation, 7, 23-31

https://doi.org/10.4236/ojapr.2019.73003

Received: May 26, 2019

Accepted: September 3, 2019

Published: September 6, 2019

Copyright (c) 2019 by author(s) and Scientific Research Publishing Inc. This work is licensed under the Creative Commons Attribution International License (CC BY 4.0).

http://creativecommons.org/licenses/by/4.0/

\begin{abstract}
An FSS based circular polarizer for high-speed wireless communication at 75 $\mathrm{GHz}$ is presented. It has been designed on a low loss substrate with cross-dipole elements. Both simulation and measured results showed more than $98 \%$ circular polarization at $75 \mathrm{GHz}$. Moreover, $3 \mathrm{~dB}$ axial-ratio bandwidth of $6.8 \mathrm{GHz}$ (Simulation) and $7.8 \mathrm{GHz}$ (Measured) has been achieved. The proposed design has many advantages over the recently published research such as simplicity, low-profile, percentage bandwidth, frequency of operation and relative insertion loss.
\end{abstract}

\section{Keywords}

Circular Polarization, Frequency Selective Surface, High Frequency

Communication, Linear Polarization

\section{Introduction}

The communication in circular polarization mode is very important in tracking and radar communication applications. It has many advantages and disadvantages to linear polarization. Some of these have lesser effect of absorption from atmosphere, multi-path fading and reflection from different sources. Moreover, in applications where the polarization is continuously changing or its tilt is not known, linear polarization cannot be used.

For inter-aircraft communications or wireless communication between mobile nodes, using circular polarization is an advantage. One way of achieving circular polarized wave communication is the use of electronically steerable antenna arrays [1] [2]. However, design and development of such systems in which electronic circuitry needs to be integrated with arrays of antennas is a challenge [3] [4]. Moreover, most of such antenna systems provide linear polarization [5] [6]. A solution to this problem is to design and develop a linear polarized an- 
tenna array and incorporate a linear to circular polarizer as a front-end. Dielectric polarizers [7], meander-line [8] [9] [10] and grid-plate polarizers [11] have been proposed to convert linear polarized electromagnetic waves to circular.

The use of FSS in many applications such as waveguides, antenna radomes, RCS reduction, wireless security, transmission improvement in energy-saving buildings and other communication applications is very popular [12]. For applications in which the linear polarization is required to convert into circular polarization as mentioned earlier, FSSs can be used to achieve this goal due its simple design and ease of fabrication [13]-[18].

This paper presents a single layer FSS linear to circular polarizer which can be used for high-frequency communication applications at $75 \mathrm{GHz}$. It is based on a simple cross-dipole FSS element. The linear to circular polarization is achieved by non-equally varying the length of dipoles in $\mathrm{x}$ and $\mathrm{y}$ directions. Both simulation and measured results show more than $98 \%$ circular polarization at 75.2 GHz. Moreover, $3 \mathrm{~dB}$ axial-ratio bandwidth of $6.8 \mathrm{GHz}$ (Simulation) and 7.8 $\mathrm{GHz}$ (Measured) is achieved.

Cross-dipole, Jerusalem cross-dipole and other structures have been used for linear to circular polarization conversion in the recent research [13]-[20], however, either these designs are for much lower frequencies with lesser percentage bandwidth or multi-layer structures adding complexity to the design. Moreover, most of these researches present simulation results only. For example in [13] a planar dual-band linear to circular polarization converter using radial-shape multi-layer FSS has been presented. Despite being dual-layer structure which adds complexity, the insertion loss is quite high and the cumulative bandwidth of both bands is not wide. Also, no measured data has been provided to validate the simulation results. Reference [14] also presents a complex design with more than $3 \mathrm{~dB}$ insertion loss which should be lower than that as expected in multi-layer designs, especially at frequencies much lower than the one presented in this paper. The FSS design presented in [16] is single layer operating at $30 \mathrm{GHz}$ with more than $4 \mathrm{~dB}$ insertion loss at the centre frequency which is quite high considering the frequency of operation. Same issues can be noticed in [16] [17] [18] [19] [20].

The proposed FSS polarizer is designed to use as a front end of a linear polarized antenna array operating at $75 \mathrm{GHz}$ for inter-aircraft communication applications. It required a simple, low-profile design having an acceptable insertion loss and high bandwidth. Both simulation and measured data are presented as well.

\section{Polarizer Design}

Figure 1 shows the unit cell of FSS polarizer. The dimensions of the length of dipoles in two directions are $1.74(\mathrm{x}-) \mathrm{mm}$ and $1.40 \mathrm{~mm}(\mathrm{y}-)$, respectively; with a width of $0.2 \mathrm{~mm}$. Rogers RT5880 having a thickness of $0.787 \mathrm{~mm}$ is used in the design [21]. Its dielectric constant is 2.2 while the loss tangent is 0.0009 . The dimension of the unit cell in both directions is $2.20 \mathrm{~mm}$. 


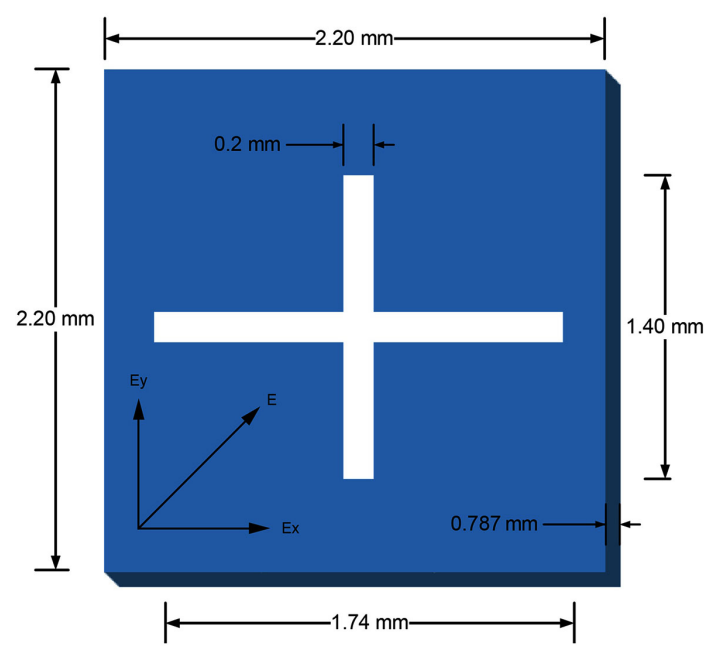

Figure 1. FSS unit cell with orientation of electrical field.

\section{FSS Simulation Setup}

CST Microwave Studio 2017 is used to setup simulation of FSS polarizer shown in Figure 1 [22]. The time domain solver with periodic boundary condition is applied to the unit cell. A plane wave port is defined with E-field tilted at $45^{\circ}$ at the side where the wave is incident on the FSS surface as shown in Figure 2. This linear polarized wave is incident with a tilt angle of $45^{\circ}$ such that it will decompose into its two orthogonal equal magnitudes in-phase components in both directions as shown in Figure 1. The Ex and Ey components are incident on the different length arms of the cross dipole. For obtaining a perfect circular polarization, there should be a $90^{\circ}$ phase difference between Ex and Ey components and their magnitude should be same. The phase difference and magnitude of E-field are obtained using CST MW Studio 2017 optimization tool [22].

Once the simulation is completed, the template-based post processing option in CST MW Studio is used to calculate the axial ratio. The following formulae have been used to calculate axial ratio [23]:

Major Axis:

$$
\sqrt{\frac{1}{2}\left[E_{x}^{2}+E_{y}^{2}+\sqrt{E_{x}^{4}+E_{y}^{4}+2 E_{x}^{2} E_{y}^{2} \cos \left(2 \delta_{L}\right)}\right]}
$$

Minor Axis:

$$
\sqrt{\frac{1}{2}\left[E_{x}^{2}+E_{y}^{2}+\sqrt{E_{x}^{4}+E_{y}^{4}+2 E_{x}^{2} E_{y}^{2} \cos \left(2 \delta_{L}\right)}\right]}
$$

where

$\delta_{L}=$ The phase difference between electric field components in $x$ - and $y$ - directions

With axial ratio given as:

$$
\mathrm{AR}=\text { Minor Axis/Major Axis }
$$

The value of axial ratio can vary from 0 to 1 . If the value of axial ratio is 1 , then it will be $100 \%$ circular polarization and for 0 it will be $100 \%$ linear. In between 0 and 1 , the polarization will tend to be elliptical. 


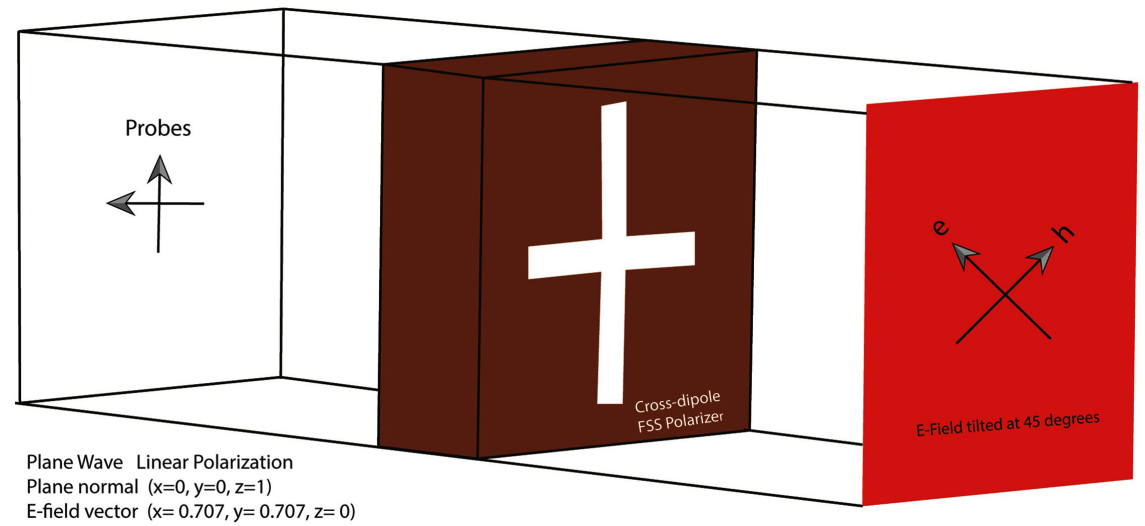

Figure 2. Plane wave excitation with E-filed oriented at $45^{\circ}$ with two probes shown in green color at the output port.

\section{Fabrication and Measurement Setup}

The FSS polarizer implementing unit cell shown in Figure 1 was fabricated and is shown in Figure 3. The dimension of the prototype is $130 \mathrm{~mm}^{2}$. The FSS consist of cross-dipoles uniformly populated within a circular area $(114 \mathrm{~mm}$ diameter). The block diagram of the measurement setup is depicted in Figure 4 while the inset shows its photograph. A pair of HP 85104A test set modules in conjunction with an HP 86105A mm-wave controller is needed with the HP 85106D Network Analyzer System to make the S-parameters measurements at mm-wave frequencies. The FSS polarizer prototype was tested using standard-gain V/W-band horn antennas as shown in Figure 4. The transmitter horn antenna which is linearly polarized (Tx) is fed from the VNA which is at a distance of 265 $\mathrm{mm}$ (V-band)/185mm (W-band) away at a normal angle to the surface of prototype. There is an equal distance of receive antenna $(\mathrm{Rx})$ from the surface of prototype. The vertical and horizontal polarizations are achieved by relative rotation of the $x-y$ aligned DUT by $90^{\circ}$. To obtain the transmission phase/magnitude in the far field, the values are normalized with respect to a similar measurement setup without the FSS polarizer array. The measurements are done in an anechoic chamber.

\section{Results}

Both measured and simulation results are shown in this section. Figure 5 shows the transmission magnitudes (in $\mathrm{dB}$ ) of Ex and Ey components of E-field. The dipoles along $\mathrm{x}$ - and y-directions resonate at $67.3 \mathrm{GHz}$ and $80.2 \mathrm{GHz}$, respectively. The two responses intersect at $75.2 \mathrm{GHz}$. Here the magnitude of both electric fields is the same, and the phase difference between them is about $89^{\circ}$. However, at frequencies above and below this frequency, the output tends to become elliptically polarized. A transmission loss of $4.8 \mathrm{~dB}$ is also noticed due to the reflection from the FSS surface. This loss can be further reduced by designing dual-layer structures using the fabry-perot concept but adds complexity to the design [23]. 


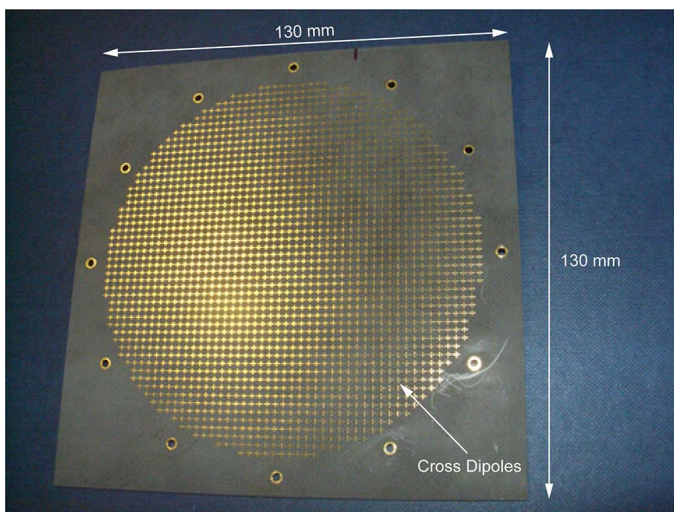

Figure 3. The photograph of the transmission polarizer with dimensions.

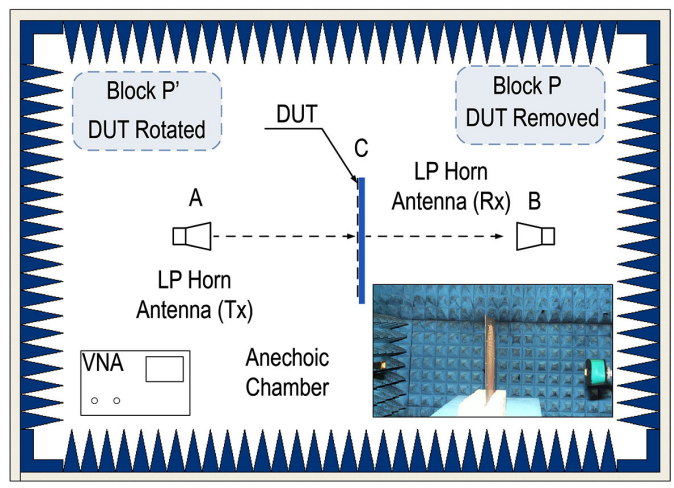

Figure 4. Block diagram of the measurement setup while its photograph is shown in inset.

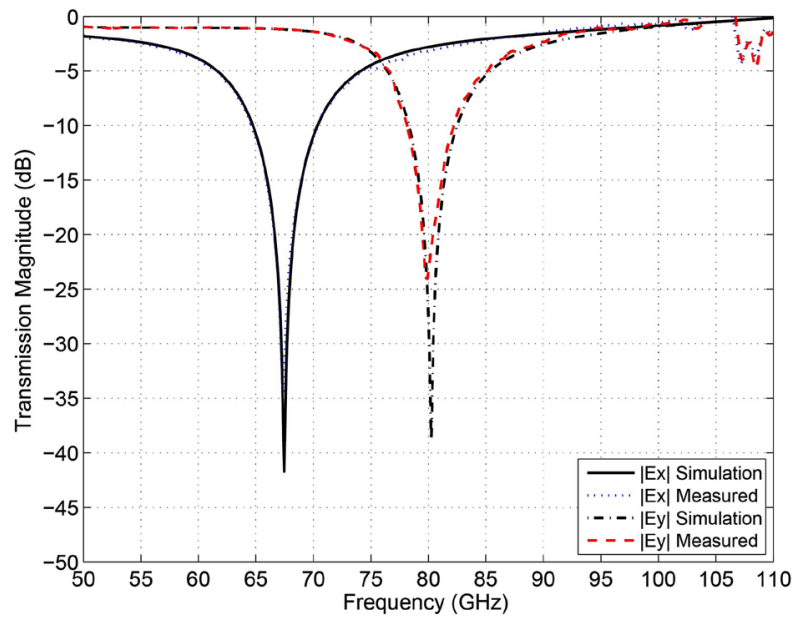

Figure 5. The Simulation and measured transmission magnitudes of Ex and Ey.

Figure 6 shows the phase of the Ex and Ey components. For Ex component, both measured and simulation results depict good agreement while for Ey component, measured results show variation at around $80 \mathrm{GHz}$. Figure 7 shows the difference in phase between two components of E-field. At $75.2 \mathrm{GHz}$, the phase 
difference is about $89^{\circ}$ giving rise to a $98 \%$ circular polarization. In simulation, the phase variation from $75.2 \mathrm{GHz}$ to $80.3 \mathrm{GHz}$ is about $87^{\circ}$ to $92^{\circ}$ which is quite close to the desired value of $90^{\circ}$. However, with phase variation, there is also a change in the magnitudes of Ex and Ey, hence giving rise to elliptical polarization as we head away from the center frequency. The phase for both simulation and measured results is same at $75.2 \mathrm{GHz}$, however, within the band of interest there is a small difference in the results which could be due to measurement inaccuracy and fabrication tolerances.

Figure 8 shows the axial ratio in $\mathrm{dB}$. In case of simulation results, a $3 \mathrm{~dB}$ bandwidth of $6.8 \mathrm{GHz}(72.2 \mathrm{GHz}$ to $78 \mathrm{GHz})$ is achieved, while the measured results show a bandwidth of $7.8 \mathrm{GHz}(67.8 \mathrm{GHz}$ to $75.6 \mathrm{GHz})$. The difference in the results is due to measurement inaccuracy in phase of Ex and Ey components. However, from $72 \mathrm{GHz}$ to $75 \mathrm{GHz}$, there is a good agreement between simulation and measured axial ratio, giving a bandwidth of $3 \mathrm{GHz}$.

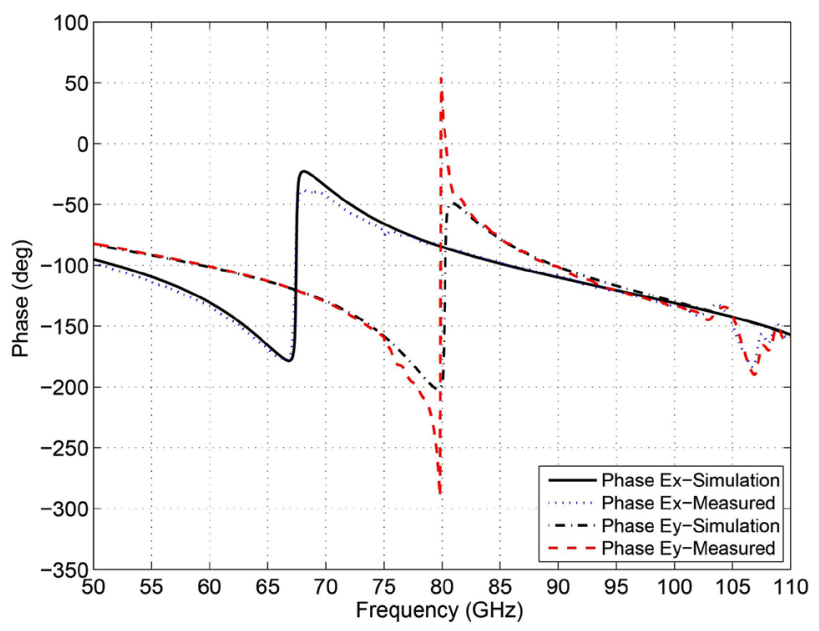

Figure 6. Simulation and measured transmission phase difference between Ex and Ey components.

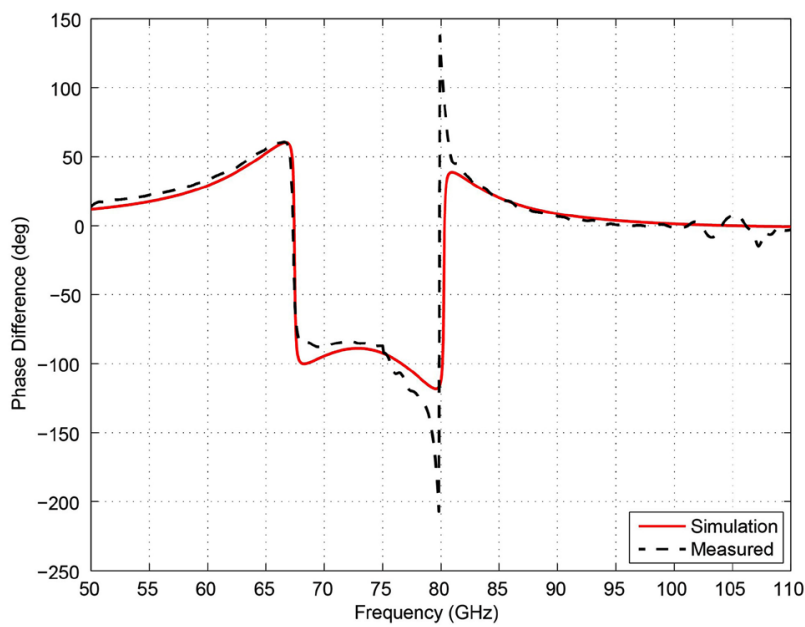

Figure 7. Simulation and measured transmission phase difference of the orthogonal components of E-field. 


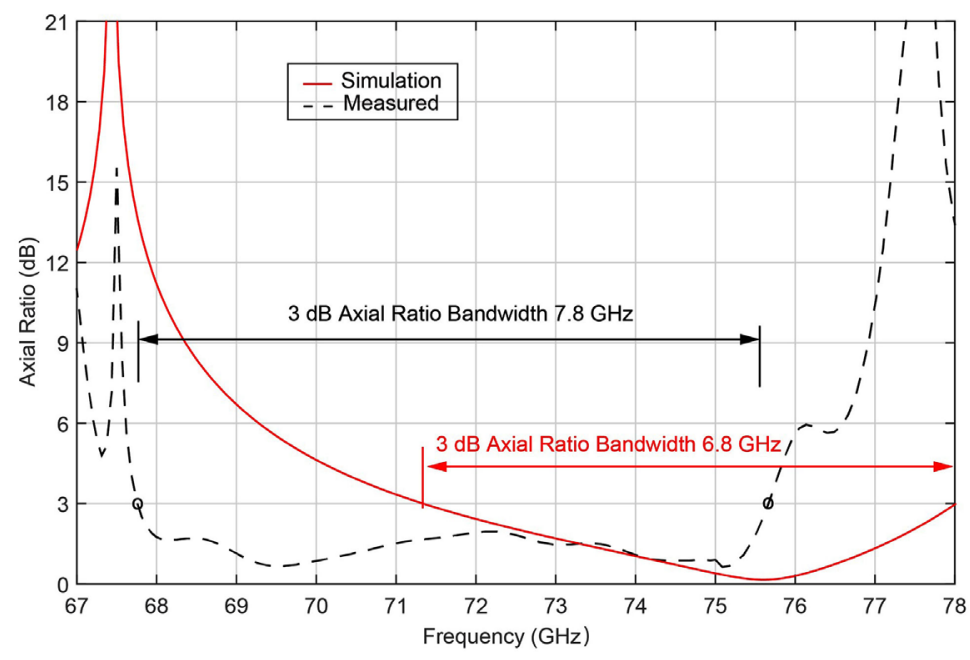

Figure 8. Simulation and measured axial ratios for transmitted E-filed components.

\section{Conclusion}

A simple FSS polarizer is presented for high-speed wireless communication at 75 GHz. The design is quite simple and can be fabricated with ease. The $3 \mathrm{~dB}$ axial ratio bandwidth of $6.8 \mathrm{GHz}(9 \%)$ and $7.8 \mathrm{GHz}(10.4 \%)$ has been achieved in simulation and measurements, respectively. It can be used as a linear to circular polarizer in inter-aircraft communication to resolve the complexity of designing circular polarized antennas as front end. Due to the reflections from polarizer surface and loss in the dielectric, there is a transmission loss of $4.8 \mathrm{~dB}$ which can be considered acceptable for a single layer polarizer operating at high frequency. The specifications of the proposed design are unique compared to the recent researches. However, research is underway to reduce the insertion loss further, achieve stable oblique incidence performance and improve $3 \mathrm{~dB}$ axial ratio bandwidth. Different FSS elements and low-loss substrates are being investigated.

\section{Acknowledgements}

The author would like to thank Prof. Vincent Fusco, Queen's University Belfast, UK, for his help in measurements and Val Dyadyuk of CSIRO Australia to provide constructive feedback on the topic.

\section{Conflicts of Interest}

The author declares no conflicts of interest regarding the publication of this paper.

\section{References}

[1] Guo, J., Huang, X. and Dyaduk, V. (2012) A Hybrid Adaptive Antenna Array for Long Range mm-Wave Communications. IEEE Antennas and Propagation Magazine, 54, 271-282. https://doi.org/10.1109/MAP.2012.6230773 
[2] Dyadyuk, V., et al. (2011) Adaptive Antenna Arrays for Ad-Hoc Millimetre-Wave Wireless Communications. In: Advanced Trends in Wireless Communications, InTech, London, Chapter 6, 93-116. https://doi.org/10.5772/16000

[3] Uchimura, H., Shino, N. and Miyazato, K. (2005) Novel Circular Polarized Antenna Array Substrates for $60 \mathrm{GHz}$-Band. IEEE MTT-S International Microwave Symposium Digest, Long Beach, 12-17 June 2005, 1-4.

[4] Wang, S.-W., et al. (2004) A Circular Polarizer Designed with a Dielectric Septum Loading. IEEE Transactions on Microwave Theory and Techniques, 52, 1719-1723. https://doi.org/10.1109/TMTT.2004.830487

[5] Deal, W.R., Kaneda, N., Sor, J., Qian, Q.Y. and Itoh, T. (2000) A New Quasi-Yagi Antenna for Planar Active Antenna Arrays. IEEE Transactions on Microwave Theory and Techniques, 48, 910-918. https://doi.org/10.1109/22.846717

[6] Nikolic, N. and Weily, A. (2009) Printed Quasi-Yagi Antenna with Folded Dipole Driver. IEEE Antenna and Propagation Symposium, Charleston, 1-5 June 2009, 1-4. https://doi.org/10.1109/APS.2009.5171674

[7] Goldstone, L. (1979) MM Wave Transmission Polarizer. IEEE Antennas and Propagation Society International Symposium, Seattle, 18-22 June 1979, 1-4.

[8] Young, L., Robinson, L. and Hacking, C. (1973) Meander-Line Polarizer. IEEE Transactions on Antenna and Propagation, 21, 376-378.

https://doi.org/10.1109/TAP.1973.1140503

[9] Chu, R.S. and Lee, K.M. (1987) Analytical Method of a Multilayered Meander-Line Polarizer Plate with Normal and Oblique Plane-Wave Incidence. IEEE Transactions on Antenna and Propagation, 35, 652-661. https://doi.org/10.1109/TAP.1987.1144158

[10] Mazur, M. and Zieniutycz, W. (2000) Multi-Layer Meander Line Polarizer for Ku Band. 13th International Conference on Microwaves, Radar and Wireless Communication, Wroclaw, 22-24 May 2000, 78-81.

[11] Chen, Z. and Li, X. (2004) Novel Mixed Grid-Plate Circular Polarizers. 4th International Conference on Microwave and Millimeter Wave Technology, Nanjing, 19-21 August 2004, 66-69.

[12] Munk, B.A. (2000) Frequency Selective Surfaces, Theory and Design. John Wiley \& Sons Inc., Hoboken. https://doi.org/10.1002/0471723770

[13] Young, Y. and Hong, W. (2018) Planar Dual-Band Linear to Circular Polarization Converter using Radial-Shape Multi-Layer FSS. IEEE International Symposium on Antennas and Propagation \& USNCI URSI National Radio Science Meeting, Boston, MA, 8-13 July 2018, 1465-1466. https://doi.org/10.1109/APUSNCURSINRSM.2018.8608738

[14] Naseri, P., Matos, S.A., Costa, J.R., Fernandes, C.A. and Fonseca, N.J.G. (2018) Dual-Band Dual-Linear-to-Circular Polarization Converter in Transmission Mode Application to K/Ka-Band Satellite Communications. IEEE Transactions on Antennas and Propagation, 66, 7128-7137. https://doi.org/10.1109/TAP.2018.2874680

[15] Ghalyon, H.A., Akbari, M. and Sebak, A. (2017) A 30 GHz Linear-to-Circular Polarization Conversion Using Two-Layer FSS. IEEE International Symposium on Antennas and Propagation \& USNCI URSI National Radio Science Meeting, San Diego, 9-14 July 2017, 671-672. https://doi.org/10.1109/APUSNCURSINRSM.2017.8072378

[16] Akbari, M., Gupta, S., Zarbakhsh, S. and Sebak, A.R. (2016) A Linear to Circular Polarizer Based on Frequency Selective Surface Operating $30 \mathrm{GHz}$ Applications. 17 th International Symposium on Antenna Technology and Applied Electromag- 
netic, Montreal, 10-13 July 2016, 1-2.

https://doi.org/10.1109/ANTEM.2016.7550113

[17] Sohail, I., Ranga, Y., Esselle, K.P. and Hay, S.G. (2013) A Linear to Circular Polarization Converter Based on Jerusalem-Cross Frequency Selective Surface. 7 th European Conference on Antennas and Propagation, Gothenburg, 8-12 April 2013, 2141-2143.

[18] Hao, J., et al. (2007) Manipulating Electromagnetic Wave Polarization by Anisotropic Metamaterials. Physical Review Letters, 99, Article ID: 063908. https://doi.org/10.1103/PhysRevLett.99.063908

[19] Zhang, W., Li, J. and Xie, J. (2017) A Broadband Linear-to-Circular Transmission Polarizer Based on Right-Angled Frequency Selective Surfaces. International Journal of Antennas and Propagation, 2017, Article ID: 8067574.

https://doi.org/10.1155/2017/8067574

[20] Zhang, W., Li, J. and Wang, L. (2016) Broadband Circular Polarizer Based on Multilayer Gradual Frequency Selective Surfaces. International Journal of Antennas and Propagation, 2016, Article ID: 4928109. https://doi.org/10.1155/2016/4928109

[21] http://www.rogerscorp.com/index.aspx

[22] http://www.cst.com/products/csts2

[23] Goldstein, D. (2007) Polarized Light. 2nd Edition, Marcel-Dekker, New York. 